\title{
Basal fatty acid oxidation increases after recurrent low glucose in human primary astrocytes
}

\author{
Paul G. Weightman Potter ${ }^{1}$. Julia M. Vlachaki Walker ${ }^{1}$ Josephine L. Robb ${ }^{1} \cdot$ John K. Chilton ${ }^{1} \cdot$ Ritchie Williamson ${ }^{2}$. \\ Andrew D Randall ${ }^{3} \cdot$ Kate L. J. Ellacott ${ }^{1} \cdot$ Craig Beall $^{1}$
}

Received: 30 May 2018 / Accepted: 22 August 2018 / Published online: 6 October 2018

(C) The Author(s) 2018

\begin{abstract}
Aims/hypothesis Hypoglycaemia is a major barrier to good glucose control in type 1 diabetes. Frequent hypoglycaemic episodes impair awareness of subsequent hypoglycaemic bouts. Neural changes underpinning awareness of hypoglycaemia are poorly defined and molecular mechanisms by which glial cells contribute to hypoglycaemia sensing and glucose counterregulation require further investigation. The aim of the current study was to examine whether, and by what mechanism, human primary astrocyte (HPA) function was altered by acute and recurrent low glucose (RLG).

Methods To test whether glia, specifically astrocytes, could detect changes in glucose, we utilised HPA and U373 astrocytoma cells and exposed them to RLG in vitro. This allowed measurement, with high specificity and sensitivity, of RLG-associated changes in cellular metabolism. We examined changes in protein phosphorylation/expression using western blotting. Metabolic function was assessed using a Seahorse extracellular flux analyser. Immunofluorescent imaging was used to examine cell morphology and enzymatic assays were used to measure lactate release, glycogen content, intracellular ATP and nucleotide ratios. Results AMP-activated protein kinase (AMPK) was activated over a pathophysiologically relevant glucose concentration range. RLG produced an increased dependency on fatty acid oxidation for basal mitochondrial metabolism and exhibited hallmarks of mitochondrial stress, including increased proton leak and reduced coupling efficiency. Relative to glucose availability, lactate release increased during low glucose but this was not modified by RLG. Basal glucose uptake was not modified by RLG and glycogen levels were similar in control and RLG-treated cells. Mitochondrial adaptations to RLG were partially recovered by maintaining euglycaemic levels of glucose following RLG exposure.

Conclusions/interpretation Taken together, these data indicate that HPA mitochondria are altered following RLG, with a metabolic switch towards increased fatty acid oxidation, suggesting glial adaptations to RLG involve altered mitochondrial metabolism that could contribute to defective glucose counterregulation to hypoglycaemia in diabetes.
\end{abstract}

Keywords Adenosine triphosphate $\cdot$ AMP-activated protein kinase $\cdot$ Astrocyte $\cdot$ Diabetes $\cdot$ Fatty acid oxidation $\cdot$ Glia $\cdot$ Hypoglycaemia $\cdot$ Lactate $\cdot$ Low glucose $\cdot$ Mitochondrial metabolism

Electronic supplementary material The online version of this article (https://doi.org/10.1007/s00125-018-4744-6) contains peer-reviewed but unedited supplementary material, which is available to authorised users.

Craig Beall

c.beall@exeter.ac.uk

1 Institute of Biomedical and Clinical Sciences, University of Exeter Medical School, RILD Building, Barrack Road, Exeter EX2 5DW, UK

2 School of Pharmacy and Medical Sciences, University of Bradford, Bradford, UK

3 Hatherly Laboratories, Prince of Wales Road, University of Exeter, Exeter, UK

$\begin{array}{ll}\text { Abbreviations } \\ \text { ACC } & \text { Acetyl-CoA carboxylase } \\ \text { AMPK } & \text { AMP-activated protein kinase } \\ \text { CPT1 } & \text { Carnitine palmitoyltransferase 1 } \\ \text { CRR } & \text { Counterregulatory response } \\ \text { ECAR } & \text { Extracellular acidification rate } \\ \text { FAO } & \text { Fatty acid oxidation } \\ \text { FCCP } & \text { Carbonyl cyanide } \\ & \text { 4-(trifluoromethoxy)phenylhydrazone } \\ \text { GFAP } & \text { Glial fibrillary acidic protein } \\ \text { HK } & \text { Hexokinase } \\ \text { HPA } & \text { Human primary astrocyte } \\ \text { MPC } & \text { Mitochondrial pyruvate carrier }\end{array}$




\section{Research in context}

\section{What is already known about this subject?}

- Glucose counterregulation is impaired following recurrent hypoglycaemia

- Specialised glucose-sensing neurons contribute to mediation of hypoglycaemia counterregulation, and are likely to be influenced by astrocytes

- These glucose-sensing neurons become dysfunctional following recurrent hypoglycaemia

What is the key question?

- Are human primary astrocytes functionally altered by exposure to acute and recurrent low glucose levels?

What are the new findings?

- Human astrocytes respond to acute hypoglycaemia by increasing activation of the AMP-activated protein kinase pathway

- Exposure to recurrent low glucose induces adaptations to mitochondrial and glycolytic function

- Mitochondria adapt to increase their dependency on fatty acid oxidation for basal respiration to successfully maintain ATP supply

\section{How might this impact on clinical practice in the foreseeable future?}

- These data highlight a potential pathological role for glial adaptations to recurrent low glucose exposure that may contribute to defective hypoglycaemia counterregulation in diabetes

\section{6-NBDG 6-(N-(7-nitrobenz-2-oxa-1,3-diazol-4-yl) amino)-6-deoxyglucose \\ OCR Oxygen consumption rate \\ PFK $[\mathrm{P}] \quad$ Phosphofructokinase [platelet] \\ RLG Recurrent low glucose \\ VDAC Voltage-dependent anion channel \\ $\mathrm{VMH} \quad$ Ventromedial nucleus of the hypothalamus}

\section{Introduction}

Hypoglycaemia remains a major concern for people with type 1 diabetes and advanced insulin-treated type 2 diabetes. For individuals with diabetes, hypoglycaemia is a major barrier to enjoying the benefits of exercise [1,2] and is increasingly thought to increase the risk of cardiovascular events in both type 1 and type 2 diabetes [3, 4]. Individuals who experience frequent hypoglycaemia or have long-duration diabetes produce diminished counterregulatory responses (CRRs) to hypoglycaemia [5, 6], often accompanied by reduced symptom awareness, termed 'impaired hypoglycaemia awareness'.

Defective glucose CRRs to hypoglycaemia are, at least in part, mediated by changes in the function of critically important glucose-sensing neurons in the ventromedial nucleus of the hypothalamus (VMH), although glucose-sensing neurons in the hindbrain have also been implicated [7]. In addition to neurons, the brain contains at least as many cells that lack electrically excitable membranes. Of these, the largest group is the glial cells, the largest subpopulation of which are astrocytes. As well as exhibiting a range of cell-to-cell signalling pathways, astrocytes are increasingly recognised as important players in central nervous system-based diseases, such as obesity [8], Alzheimer's disease [9], Parkinson's disease [10] and motor neuron disease [11].

Astrocytes play an important role in regulating glucose uptake into cerebral microvessels [12]. In addition to this role in glucose delivery, more recent studies have demonstrated that stimuli such as 2-deoxyglucose, that block intracellular glucose metabolism, increase intracellular $\mathrm{Ca}^{2+}$ in hindbrain astrocytes [13], a well-accepted marker of increased astrocyte activity, suggesting a more direct role in sensing changes in glucose levels. In the hypothalamus, astrocyte activation by fasting is indicated by increased glial fibrillary acidic protein (GFAP) expression and process complexity $[14,15]$, both markers of gliosis.

Astrocytes contain small amounts of glycogen that can be broken down to glucose and metabolised to lactate, which is released to feed active neurons. For example, astrocytic glycogen stores can maintain axonal activity during energetic stress in white matter $[16,17]$ and also contribute to memory formation $[18,19]$. Brain glycogen concentrations are increased on recovery from exercise [20], suggesting that astrocytic glycogen stores are both physiologically and pathophysiologically regulated. Astrocytes are in intimate apposition to the cerebral vasculature, meaning that a change in glycaemia may be detected by astrocytes before neurons. Moreover, astrocytes are generally accepted as metabolically flexible neural cells that can rapidly 
upregulate glycolysis to support neuronal function, partly through the higher expression of 6-phosphofructo-2-kinase type 3 (PFKFB3), which can stimulate glycolysis [21]. Despite this, little is known about the intrinsic response of astrocytes to acute and recurrent low glucose (RLG) that occurs in type 1 diabetes.

Given that astrocytes are metabolically flexible cells, we hypothesised that human astrocytes would: (1) sense low glucose by increasing phosphorylation of cellular energy sensor AMP-activated protein kinase (AMPK); and (2) exhibit altered glycolytic and mitochondrial metabolism following RLG. To test this, we utilised both human primary astrocytes (HPA) and U373 astrocytoma cells and measured directly components of glycolytic and mitochondrial metabolism, lactate release, glycogen levels, intracellular nucleotide levels and expression of key metabolic enzymes.

\section{Methods}

Astrocyte isolation and cell culture HPA were isolated from a 17 year old male donor from subventricular deep white matter tissue immediately post-mortem following consent from next of-kin and with ethical approval from the North and East Devon Research Ethics Committee. Astrocytes were isolated from tissue blocks as previously described [22], subcultured and frozen stocks prepared. HPA cells were maintained in humidified incubators with $95 \% \mathrm{O}_{2} / 5 \% \mathrm{CO}_{2}$ in HPA stock media. U373 cells were obtained from American Type Culture Collection (ATCC, Manassas, VA, USA). See electronic supplementary material (ESM) Methods for further details of stock media components.

For experiments, the glucose concentration was reduced to $2.5 \mathrm{mmol} / \mathrm{l}$ glucose for $2 \mathrm{~h}$, supplemented with $1 \%$ serum and $22.5 \mathrm{mmol} / \mathrm{l}$ mannitol for HPA cells. Glucose concentrations were then dropped further to $0.1 \mathrm{mmol} / 1$ glucose as a hypoglycaemic-like stimulus or plated at $2.5 \mathrm{mmol} / \mathrm{l}$ glucose, supplemented with $1 \%$ (vol./vol.) serum and $24.9 \mathrm{mmol} / \mathrm{l}$ or $22.5 \mathrm{mmol} / \mathrm{l}$ mannitol, for HPA and U373 cells, respectively. For RLG studies over 4 days, control cells were exposed to zero bouts $(2.5 \mathrm{mmol} / \mathrm{l}$ glucose instead) and RLG cells exposed to 4 bouts of $3 \mathrm{~h}$ of $0.1 \mathrm{mmol} / 1$ glucose and recovered in media containing high (HPA, $5.5 \mathrm{mmol} / \mathrm{l} ; \mathrm{U} 373,7.5 \mathrm{mmol} / \mathrm{l}$ ) glucose and FBS overnight (see ESM Fig. 1 for culture model and ESM Methods for further details). The reasons for this were twofold: (1) higher glucose levels overnight (approximately $19 \mathrm{~h}$ ) prevented glucose depletion from the media, which would occur if the cells were maintained at $2.5 \mathrm{mmol} / \mathrm{l}$; and (2) this pattern more accurately represented a model of glucose variation seen in type 1 diabetes.

All cell lines used were verified as mycoplasma-free using a commercial test kit (MycoAlert, Lonza, Slough, UK). Cells were randomly assigned to experimental groups by an unblinded investigator (unless otherwise stated).
Immunoblotting Cellular protein was isolated as previously described [23], with minor modifications. Briefly, for acute studies $4.5 \times 10^{5}$ cells or, for RLG studies, $2.5 \times 10^{5}$ cells were seeded on to $60 \mathrm{~mm}$ petri dishes and harvested in $65 \mu \mathrm{l}$ lysis buffer. Protein concentrations were assessed by Bradford assay. A $10 \mu \mathrm{g}$ sample of protein was loaded per lane on 7-10\% (wt/vol.) polyacrylamide gels. Proteins were transferred to nitrocellulose membranes, blocked with BSA ( $5 \% \mathrm{wt} / \mathrm{vol}$.) or powdered milk (5\% wt/vol.) and probed with antibodies against target proteins. See ESM Methods for antibody details.

Analysis of cellular metabolism HPA and U373 cells were seeded at $2 \times 10^{4}$ cells per well in Seahorse $\mathrm{XF}^{\mathrm{e}} 96$ assay plates (no. 102416-100, Agilent, Craven Arms, UK) on the day before the study. Medium was exchanged for low-buffered media, the $\mathrm{pH}$ adjusted to 7.40 at $37.0^{\circ} \mathrm{C}$ and cells placed in an atmospheric $\mathrm{CO}_{2}$ incubator, to remove $\mathrm{CO}_{2}$ buffering capacity, $1 \mathrm{~h}$ prior to starting the assay. Mitochondrial stress tests (no. 103015-100, Agilent) and mitochondrial fuel flexibility tests (no. 103270-100, Agilent) were performed as per manufacturer's instructions (see ESM Methods for further details). Samples with negative values for OCR (probably due to the presence of an air bubble) were excluded from analysis.

Measurement of intracellular nucleotides Total ATP levels were measured using ATPlite (no. 6016941, Perkin Elmer, Seer Green, UK) with minor modifications as previously described [23]. ATP/ADP ratios were assayed by luminescence assay (no. MAK135, Sigma-Aldrich, Gillingham, UK). Briefly, $1 \times 10^{3}$ cells were seeded on to black-walled 96 well plates and exposed to $2.5 \mathrm{mmol} / 1$ or $0.1 \mathrm{mmol} / 1$ glucose levels for 15-180 min and nucleotide ratios assayed as per manufacturer's instructions.

Measurement of extracellular lactate, glucose uptake and glycogen levels Extracellular lactate was measured by assessing $\mathrm{NADH}$ production from $\mathrm{NAD}^{+}$in the presence of lactate dehydrogenase as previously described [24]. Briefly, $100 \mu$ of extracellular supernatant fraction was examined against a standard curve of lactate from $0 \mathrm{nmol} / 1$ to $50 \mathrm{nmol} / 1$. Glucose uptake was measured using fluorescently labelled glucose analogue 6-( $N$-(7-nitrobenz-2-oxa-1,3-diazol-4yl)amino)-6-deoxyglucose (6-NBDG; CAY13961-5/6NBDG, Cayman Chemical, Tallinn, Estonia). 6-NBDG $(600 \mu \mathrm{mol} / \mathrm{l})$ was added for $15 \mathrm{~min}$ in $2.5 \mathrm{mmol} / \mathrm{l}$ glucose in control conditions and in cells exposed to RLG. Glycogen levels were measured using a fluorometric kit as per manufacturer's instructions (no. MET-5023, Cambridge Bioscience, Cambridge, UK).

Fluorescent imaging Mitochondrial morphology was examined by staining HPA cells with MitoTracker Red CMXRos (50 nmol/1; M7512, no. 1785958, Thermo Fisher Scientific, 
Loughborough, UK) before fixing and imaging using confocal microscopy (Leica, London, UK DMi8; $\times 63$ /oil immersion lens) by an investigator blind to sample identity. See ESM Methods for further details. To examine intermediate filament protein expression/localisation, HPA cells were fixed and stained with an anti-vimentin antibody (V6630, no. 102M4831, mouse, Sigma-Aldrich; 1:500 in lysine buffer) or an anti-GFAP antibody (z0334 no. 20035994, rabbit, Agilent; 1:100 in lysine buffer). All antibodies were validated by the manufacturer. See ESM Methods for further details.

Statistical analysis For immunoblotting, densitometric values were normalised to unity to examine relative fold change in expression. A one-sample $t$ test was used to determine significant changes in phosphorylation or expression, relative to control. For comparisons of two groups a two-tailed unpaired $t$ test was used and for multiple group comparisons, a one-way ANOVA with post hoc Bonferroni were used. To compare the mean differences between groups split by two independent variables, a two-way ANOVA with Bonferroni multiple comparisons tests was used. Statistical tests were performed using GraphPad Prism software (Prism 5; GraphPad Software, La Jolla, CA, USA). Results are expressed as mean \pm standard error, unless otherwise stated. Values of $p<0.05$ were considered statistically significant.

\section{Results}

AMPK is activated by acute low glucose in human astrocytes AMPK activation in neurons is required for sensing hypoglycaemia [25], though it is not known whether astrocytic AMPK reacts to the same energy stress. To address this, we exposed human astrocytes to normal $(2.5 \mathrm{mmol} / \mathrm{l})$ and low $(0.1 \mathrm{mmol} / \mathrm{l})$ glucose levels for brain cells. In cells exposed to low glucose for $30 \mathrm{~min}$, we noted, in both the HPA (Fig. 1a, c) and U373 cells (Fig. 1b, d), an increased phosphorylation of AMPK at threonine 172, a site which must be phosphorylated for full kinase activation. This was accompanied by increased phosphorylation of acetyl-CoA carboxylase (ACC) at serine 79 (Fig. 1a, b, e, f). These novel observations in glia are consistent with the ubiquitous role of AMPK in detecting low glucose levels in other cells types. In contrast, although the astrogliosis marker GFAP was expressed in HPA cells, its expression appeared unaltered in cells exposed to acute low glucose (ESM Fig. 2).

\section{RLG exposure increases mitochondrial metabolism in human} astrocytes To determine whether baseline metabolism was altered following RLG, we performed mitochondrial stress tests on HPA and U373 cells (see ESM Fig. 1 for RLG model). In order to observe any cellular adaptations, this was

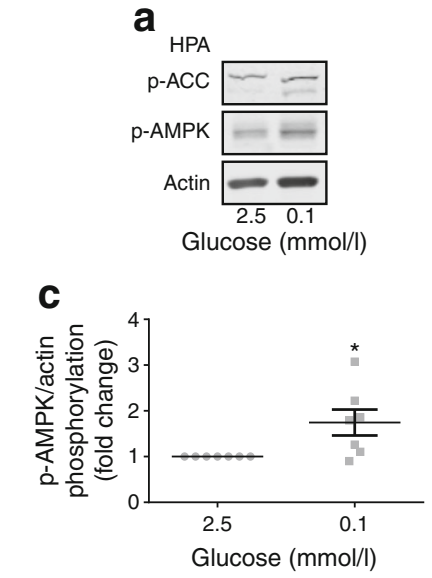

b
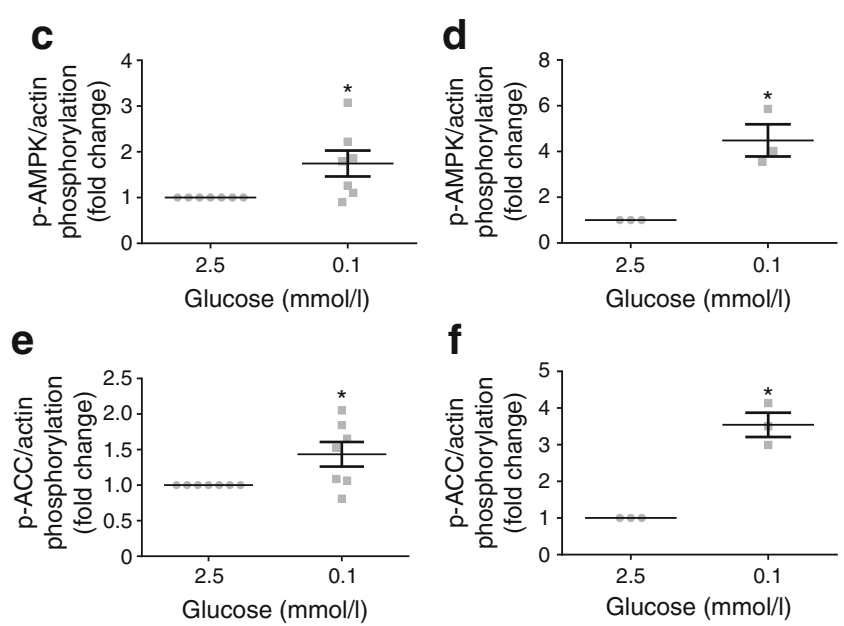

Fig. 1 Acute low glucose increased AMPK pathway activation. Representative immunoblots from HPA $(\mathbf{a} ; n=8)$ and U373 astrocytoma cells $(\mathbf{b} ; n=3)$ exposed to 2.5 or $0.1 \mathrm{mmol} / 1$ glucose media for $30 \mathrm{~min}$. (c), d) Densitometric analysis of immunostaining for pT172 AMPK normalised to actin as a loading control in HPA (c) and U373 astrocytoma cells (d). (e, f) Densitometric analysis of immunostaining for pS79 ACC normalised to actin as a loading control in HPA (e) and U373 astrocytoma cells $(\mathbf{f})$. Error bars represent SEM. * $p<0.05$, one-sample $t$ test vs control ( $2.5 \mathrm{mmol} / \mathrm{l}$ glucose)

performed in $2.5 \mathrm{mmol} / \mathrm{l}$ glucose, representing euglycaemic, glucose levels following RLG.

First, we observed significantly higher baseline oxygen consumption rate (OCR) in both HPA (Fig. 2a, c) and U373 cells (Fig. $2 b, d$ ) exposed to RLG ( 3 vs 0 prior bouts of low glucose), indicating persistent adaptations in cellular metabolism. Oligomycin was then added to block ATP synthase, carbonyl cyanide 4-(trifluoromethoxy)phenylhydrazone (FCCP) to stimulate maximal respiration, and rotenone and antimycin A were added to inhibit complex I and III, respectively. This allowed calculation of coupling efficiency, which was significantly decreased in HPA cells only (Fig. 2e, f). However, both cell types displayed increased proton leak (Fig. $2 \mathrm{~g}, \mathrm{~h}$ ), indicating mitochondrial dysfunction. Despite the evidence of mitochondrial dysfunction, we saw no changes in the morphology of the filamentous mitochondrial networks between the treatment groups, as examined using MitoTrackerRed (ESM Fig. 3a, b).

In addition to changes in OCR, we also noted significantly elevated basal extracellular acidification rate (ECAR), in HPA cells exposed to RLG (Fig. 2i, k), indicating enhanced glycolysis following RLG. This did not occur within U373 cells following RLG (Fig. 2j, 1). 

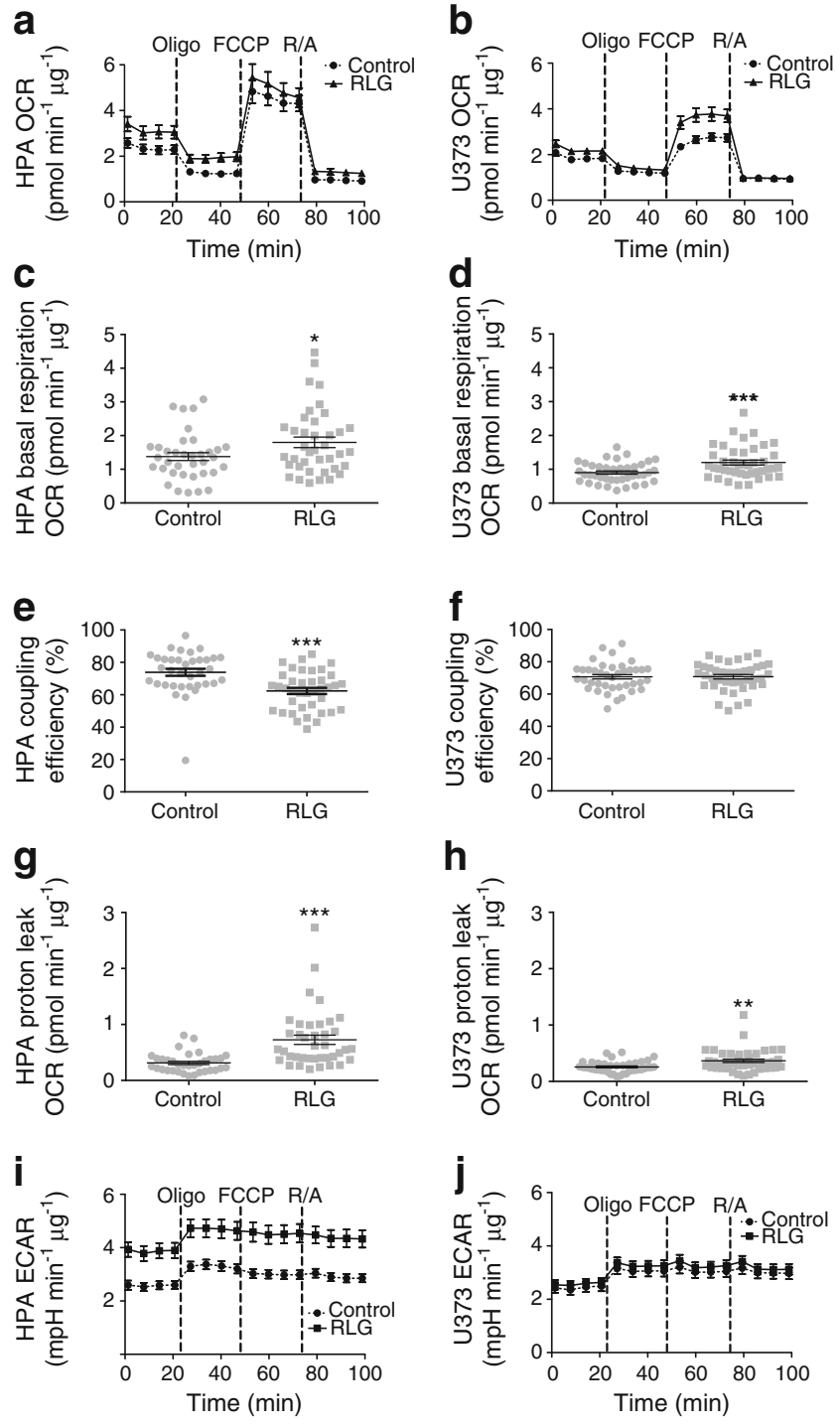

k
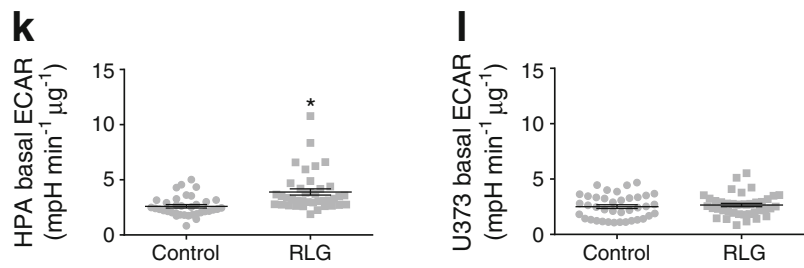

Fig. 2 RLG increased baseline mitochondrial metabolism and reduced mitochondrial coupling efficiency in HPA (a,c,e,g,i,k) and U373 astrocytoma cells (b,d,f,h,j,l). (a, b) OCR of HPA (a; control, $n=36$; RLG, $n=$ 39 , across three separate assays) and U373 cells (b; control, $n=40$; RLG, $n=43$, across three separate assays) following RLG. Cells were exposed to oligomycin $(10 \mu \mathrm{mol} / \mathrm{l})$, FCCP $(5 \mu \mathrm{mol} / \mathrm{l})$ and a combination of rotenone and antimycin A $(5 \mu \mathrm{mol} / \mathrm{l})$. (c, d) Mean basal OCR in control and RLG-treated astrocytes. (e, f) Coupling efficiency calculated from the ratio of oligomycin-sensitive OCR and basal OCR expressed as a percentage. $(\mathbf{g}, \mathbf{h})$ Proton leak, calculated from the oligomycin-insensitive (i.e. not ATP synthase linked) OCR minus non-mitochondrial respiration. (i, j) ECAR analysis measured during mitochondrial stress tests. (k, l) Mean baseline ECAR following RLG. Error bars represent SEM. ${ }^{*} p<0.05, * * p<0.01$ and $* * * p<0.001$, unpaired two-tailed student's $t$ test vs control. $\mathrm{mpH}$, milli-pH; Oligo, oligomycin; R/A, rotenone and antimycin $\mathrm{A}$
Astrocytes exposed to RLG display enhanced glycolysis on recovery of glucose level Given the increased basal ECAR in HPA cells exposed to RLG, we next examined glycolytic re-activation after an acute glucose withdrawal ( $\sim 80 \mathrm{~min}$ ) in HPA cells following prior exposure to RLG. Under control conditions, we noted that the ECAR (glycolysis) response to glucose was concentration dependent (Fig. 3a-c), although ECAR was not significantly higher at $5.5 \mathrm{mmol} / \mathrm{l}$ glucose than $2.5 \mathrm{mmol} / 1$ glucose, indicating that the metabolic response to glucose is saturated at physiological glucose levels. Interestingly, the ECAR response to glucose in cells exposed to RLG (three bouts of low glucose) was significantly greater than control (no bouts of low glucose), indicating an enhanced glycolytic capacity (Fig. 3a-c). This only occurred at euglycaemic and hyperglycaemic levels. Consistent with enhanced glycolytic activity, we also noted a significant reduction in OCR following glucose injection, indicative of a shift away from mitochondrial metabolism towards glycolysis (Fig. 3d-f). Correspondingly, the reduction in OCR following glucose recovery was augmented in HPA cells exposed to RLG. Taken together, these data suggest an enhanced Warburg-like effect in HPA cells following recovery of glucose levels. In U373 cells, RLG did not alter ECAR between groups (control vs RLG) on glucose recovery. However, the re-activation of ECAR on delivery of glucose was not concentration dependent and in U373 cells the relative increase in ECAR was twice as large as that in HPA cells (ESM Fig. 4). These studies highlight clear differences between primary cells, obtained from source, with those of transformed cells which should be taken into consideration when designing experiments.

Fatty acid oxidation is increased in human astrocytes following RLG To assess potential changes to mitochondrial fuel usage following RLG, we performed mitochondrial fuel flexibility tests, achieved by sequential selective inhibition of mitochondrial pyruvate carrier (MPC; glucose oxidation pathway), glutaminase (glutamine oxidation pathway) and carnitine palmitoyltransferase 1 (CPT1; fatty acid oxidation pathway). Surprisingly, there was no change in mitochondrial dependency, capacity or flexibility to metabolise pyruvate in HPA cells (Fig. 4a-c). This suggests that in astrocytes, alterations to glucose metabolism are upstream of the transport of pyruvate into the mitochondria. There was a non-significant trend towards reduced glutamine dependency at baseline following RLG (Fig. 4d), with no significant change to the glutamine oxidation capacity (Fig. 4e). Combined, this produced a significant increase in glutamine flexibility (Fig. 4f). Interestingly, RLG significantly increased metabolic dependency on fatty acids but with no change in fatty acid oxidation (FAO) maximum capacity, thereby causing a decrease in flexibility (Fig. 4g-i). These data demonstrate that RLG induces 
Fig. 3 RLG increased glycolytic re-activation after acute low glucose exposure. $(\mathbf{a}-\mathbf{c})$ ECAR increased in HPA cells on reintroduction of $0.5 \mathrm{mmol} / 1$ glucose (a), $2.5 \mathrm{mmol} / 1$ glucose (b) and $5.5 \mathrm{mmol} / 1$ glucose (c). (d-f) OCR decreased on addition of $0.5 \mathrm{mmol} / 1$ glucose $(\mathbf{d})$, $2.5 \mathrm{mmol} / 1$ glucose (e) and $5.5 \mathrm{mmol} / \mathrm{l}$ glucose (f). Dashed line, control; solid line, RLG. Control, $n=8-10$; RLG, $n=9$ 10. Error bars represent SEM. $* p<0.05 ; * * p<0.01$ and $* * * p<0.001$, unpaired two-tailed student's $t$ test vs control. Glu, glucose; $\mathrm{mpH}$, milli-pH

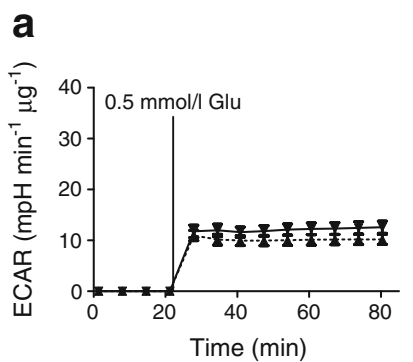

b

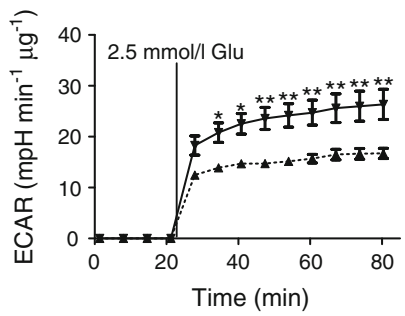

d

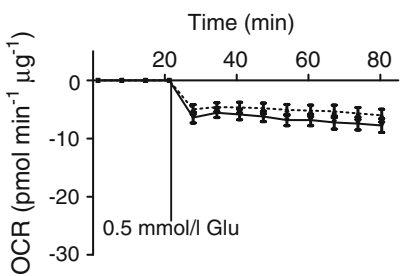

\section{e}

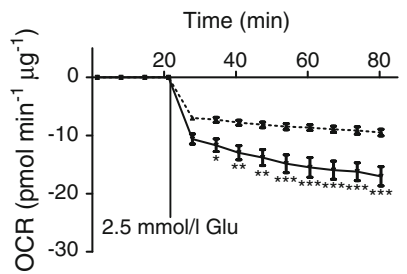

C

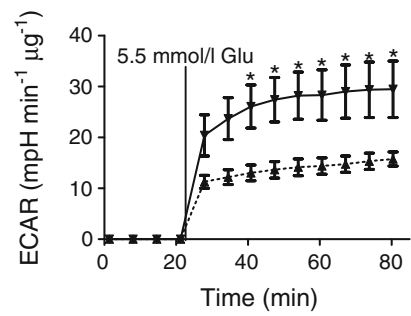

f

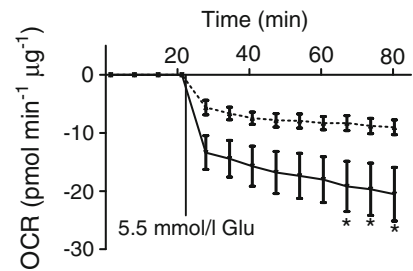

mitochondrial adaptations to fuel sources, consistent with a shift towards increased reliance on FAO and an enhanced flexibility to utilise glutamine.

Human astrocytes maintain energy production (total ATP and ATP/ADP ratio) during low glucose conditions To examine the consequences of acute low glucose and RLG for astrocyte energy content, we measured total ATP and ATP/ADP ratios. These measurements were made both in the presence of acute low glucose and following RLG. Interestingly, total ATP levels did not significantly change in HPA (Fig. 5a) or U373 cells (Fig. $5 b$ ) during acute low glucose exposure $(0.1 \mathrm{mmol} / \mathrm{l})$
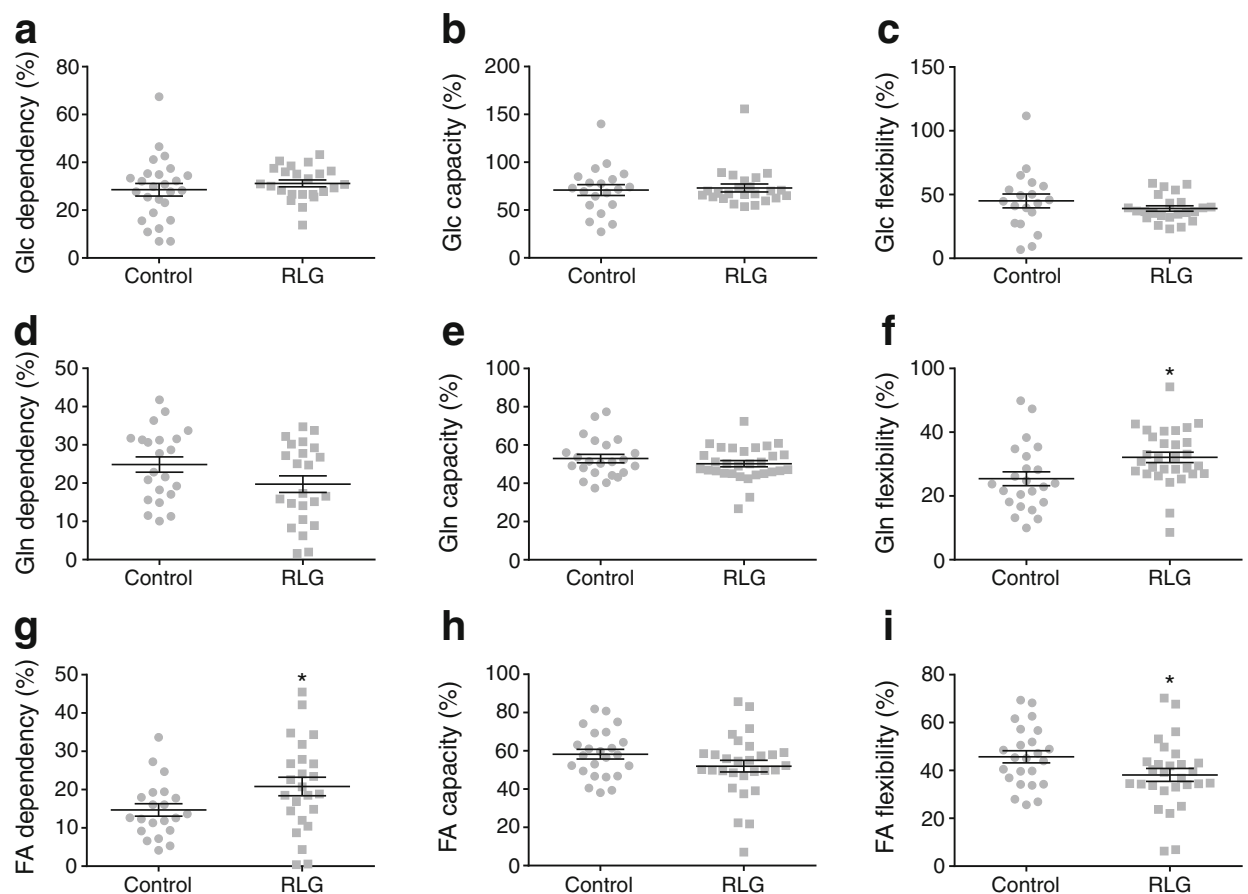

Fig. 4 RLG altered HPA mitochondrial fuel flexibility to increase dependency on fatty acids for basal oxidation. (a-c) Inhibition of MPC using UK5099 $(2 \mu \mathrm{mol} / \mathrm{l})$ to measure dependency (a), maximum capacity (b) and flexibility (c) within the glucose oxidation pathway. (d-f) Inhibition of glutaminase using BPTES (bis-2-(5-phenylacetamido-1,3,4thiadiazol-2yl)ethyl sulfide; $3 \mu \mathrm{mol} / \mathrm{l})$ to measure dependency (d), maximum capacity (e) and flexibility (f) in the glutamine oxidation pathway following RLG. (g-i) Inhibition of CPT1 using etomoxir $(4 \mu \mathrm{mol} / \mathrm{l})$ to

measure dependency (g), maximum capacity (h) and flexibility (i) in the FAO pathway following RLG. Dependency: contribution of that pathway to baseline OCR. Capacity: maximum ability to oxidise through that pathway when two other oxidative pathways are inhibited. Flexibility: calculated from the difference between dependency and capacity. Control, $n=21$; RLG, $n=24$. Error bars represent SEM. * $p<0.05$, unpaired two-tailed Student's $t$ test vs control. FA, fatty acid; Glc, glucose; Gln, glutamine 
Fig. 5 Acute and recurrent low glucose did not modify intracellular ATP or ATP/ADP ratios. $(\mathbf{a}, \mathbf{b})$ Total intracellular ATP levels in HPA $(\mathbf{a}, n=6)$ and U373 astrocytoma cells $(\mathbf{b} ; n=6)$ exposed to different glucose concentrations for $3 \mathrm{~h}$. (c, d) Intracellular ATP/ADP ratios in HPA $(\mathbf{c} ; n=6)$ and U373 astrocytoma cells $(\mathbf{d} ; n=4)$ exposed to 2.5 or $0.1 \mathrm{mmol} / 1$ glucose for $3 \mathrm{~h}$. One-way ANOVA with post hoc Bonferroni multiple comparisons tests. Glu, glucose; tATP, total ATP a

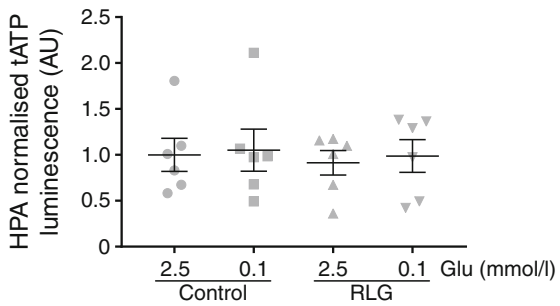

C

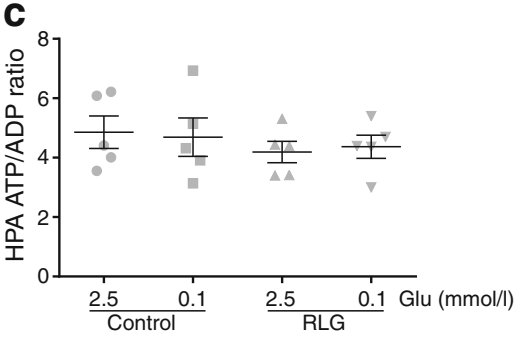

b
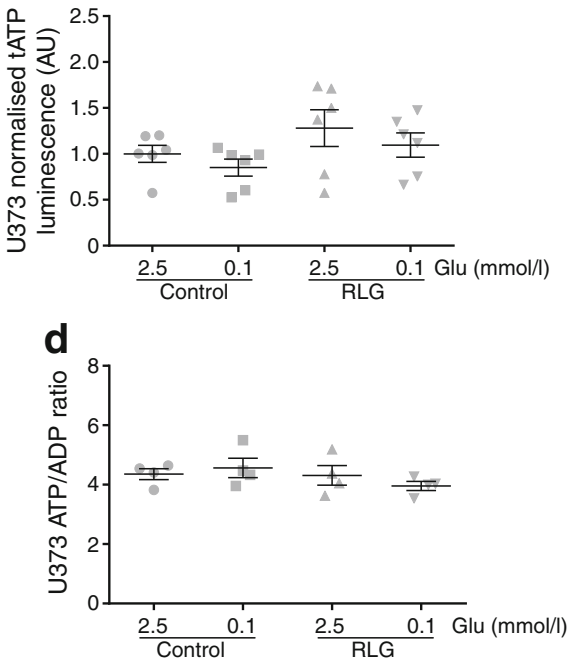

lasting $3 \mathrm{~h}$. Despite the increased basal mitochondrial and glycolytic metabolism described above, total ATP levels following RLG were also comparable with control (Fig. 5a, b). Similarly, the ATP/ADP ratio was not significantly different following $3 \mathrm{~h}$ of $0.1 \mathrm{mmol} / 1$ glucose, nor was the basal ratio altered following RLG (Fig. 5c, d). These data indicate that the total ATP content and ATP/ADP ratio are well maintained in astrocytes, at least in response to up to $3 \mathrm{~h}$ of low glucose, and that changes to glycolytic and mitochondrial metabolism may be compensatory to sustain intracellular nucleotide levels.

RLG does not alter glucose uptake or glycogen levels in human astrocytes Western blotting demonstrated that the levels of key glycolytic markers GLUT1, hexokinase (HK)I, HKII, HKIII and phosphofructokinase [platelet] (PFK[P]) were not altered by acute or RLG in human astrocytes (ESM Fig. 5). Lactate release modestly decreased from HPA cells and significantly decreased from U373 cells during $3 \mathrm{~h}$ of low glucose exposure (Fig. 6a, b), although the decrease was proportionally less than the 25 -fold reduction in glucose availability. Hence, when corrected for glucose availability, there was a relative increase in lactate release (Fig. 6c, d); however, this was not different between control and RLG-treated cells.

We next assessed the degree of glucose uptake by human astrocytes using the fluorescently labelled glucose analogue, 6-NBDG. HPA cells exposed to RLG showed no change in 6NBDG uptake (Fig. 6e), indicating that the persistent changes in mitochondrial oxygen consumption were not simply mediated by increased glucose uptake. Correspondingly, we did not observe any changes in astrocytic glycogen content following RLG (Fig. 6f).

Correlating with our mitochondrial imaging studies, the levels of mitochondrial markers succinate dehydrogenase (SDH) and the voltage-dependent anion channel (VDAC) were not altered by RLG, suggesting no change in total mitochondrial content (ESM Fig. 5). Fatty acid synthase (FAS) and CPT-1a expression were also not altered, indicating that changes in fatty acid-dependency following RLG are likely to be mediated by metabolic flux/activity rather than alternations in expression of enzymes within the FAO pathway.

Partial recovery of metabolic changes by maintenance of glucose levels To assess whether mitochondrial changes induced by RLG were reversible, we exposed cells to RLG and introduced a 4 day recovery period where glucose levels were maintained at $2.5 \mathrm{mmol} / \mathrm{l}$ glucose following the last bout of low glucose exposure. As previously, our RLG treatment group had elevated basal OCR, increased proton leak and elevated basal ECAR (Fig. 7). Following recovery, basal mitochondrial OCR returned to levels comparable with control (Fig. 7a, b). In contrast, coupling efficiency was still reduced (Fig. 7c) and proton leak showed a trend towards recovery, which did not reach statistical significance (Fig. 7d). RLGinduced elevated basal ECAR was largely unchanged following recovery (Fig. 7e, f).

\section{Discussion}

Our studies shed new light on the previously uncharacterised intrinsic metabolic changes in human glial cells in response to acute and recurrent low glucose. We demonstrate that astrocytes, in the absence of physical and chemical signals from neurons, react to low glucose by increasing the activation of the key metabolic sensor AMPK. Changes in AMPK pathway phosphorylation occur within a pathophysiologically relevant glucose concentration range, similar to that which affects CNS glucose-sensing neurons [26]. 
Fig. 6 RLG does not alter lactate release, glucose uptake or glycogen levels in HPA. $(\mathbf{a}, \mathbf{b})$ Extracellular lactate levels measured from conditioned media from HPA $(\mathbf{a} ; n=8)$ and $\mathrm{U} 373(\mathbf{b} ; n=6)$ following exposure to 2.5 or $0.1 \mathrm{mmol} / 1$ glucose-containing media for $3 \mathrm{~h}$. (c, d) Extracellular lactate levels normalised to glucose availability from conditioned media from HPA $(\mathbf{c} ; n=8)$ and $\mathrm{U} 373(\mathbf{d} ; n=$ 6) following exposure to 2.5 or $0.1 \mathrm{mmol} / 1$ glucose-containing media for $3 \mathrm{~h}$. (e) Fluorescent signal from labelled glucose analogue 6-NBDG incubated with HPA cells for $15 \mathrm{~min}$. (f) Glycogen content of HPA cells in control and following exposure to $\operatorname{RLG}(n=5) . * p<0.05$, $* * p<0.01$ and $* * * p<0.001$, one-way ANOVA with post hoc Bonferroni multiple comparisons tests, and unpaired two-tailed Student's $t$ test. RFU, relative fluorescence units a

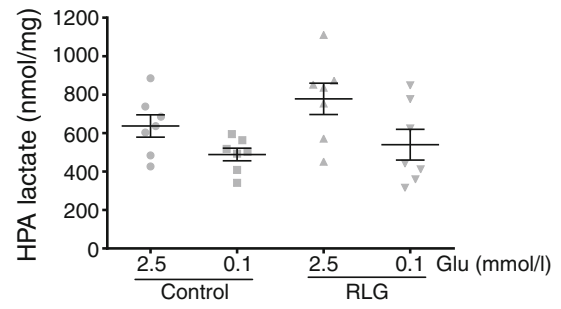

C

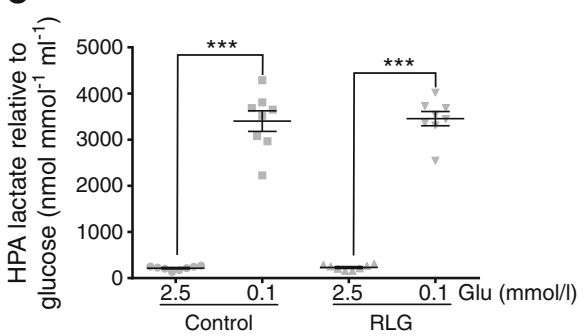

e

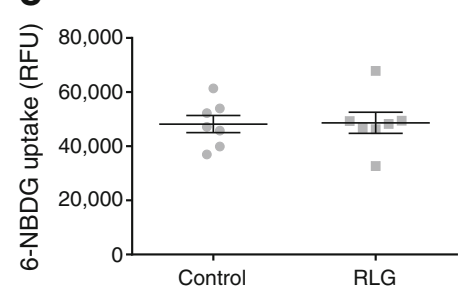

b

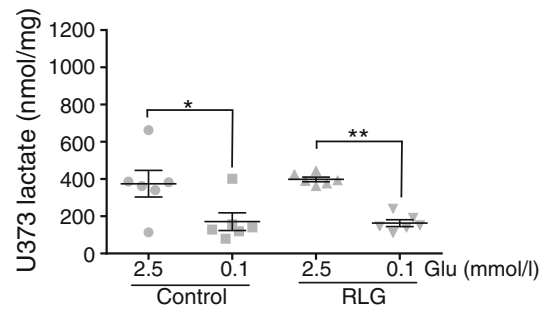

d

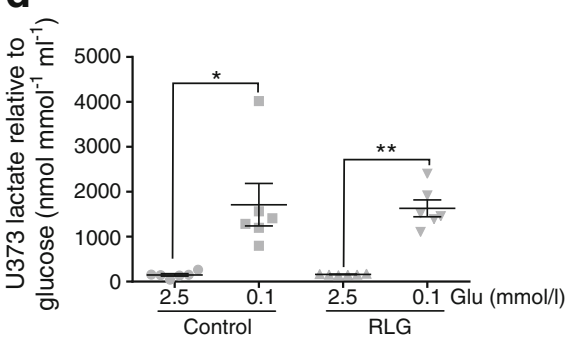

f

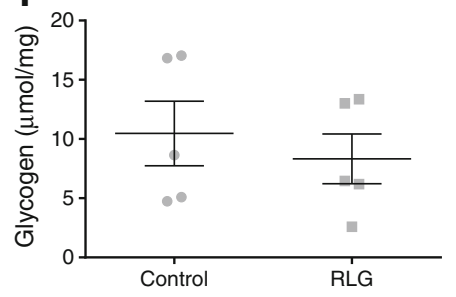

It is well accepted that astrocytes are more metabolically flexible than neurons, partly because of the higher expression of PFKFB3, which can accelerate glycolysis during metabolic stress [21]. Therefore, to examine specifically the changes in astrocyte metabolism following RLG, we measured markers of mitochondrial and glycolytic metabolism in HPA monocultures. Importantly, we found that daily prior bouts of low glucose $(4 \times 0.1 \mathrm{mmol} / 1$ glucose $)$, lasting $3 \mathrm{~h}$, were sufficient to increase basal mitochondrial and glycolytic metabolism to sustain intracellular ATP supply. Increased basal mitochondrial metabolism is likely mediated, at least in part, by an increased contribution of FAO to basal oxygen consumption. Correlating with this hypothesis, we noted increased mitochondrial proton leak and reduced mitochondrial coupling efficiency in astrocytes exposed to RLG. Importantly, FAO is known to increase uncoupling, as FAO generates increased levels of reactive oxygen species (ROS)/superoxide [27] that can increase proton leak via activation of uncoupling proteins or the ATP/ADP antiporter (ANT; [28]). Previous work has demonstrated that, in vivo, genes for $\beta$-oxidation are induced by acute hypoglycaemia, but this fails following recurrent hypoglycaemia [29]. Our data in astrocyte monocultures contrast with these measurements taken from rodent mediobasal hypothalamus, which include both neurons and glia and axon projections tracking through the dissected area. Therefore, it is possible that cell-type-specific changes are masked when measuring mRNA expression from whole-tissue sections.

A previous study demonstrated that low intracellular glucose levels caused by loss of insulin receptors on astrocytes (leading to reduced astrocytic glucose transport) resulted in astrocytic mitochondria that were smaller and less numerous [30], suggesting increased mitophagy. In our study, despite evidence of mitochondrial stress and in contrast to our expectation, examination of the filamentous mitochondrial networks revealed no change in either mitochondrial size or number following acute low glucose or RLG. Indeed, mitochondria exposed to RLG generally displayed a normal filamentous network, with little or no evidence of mitochondrial fragmentation. These data suggest that astrocyte mitochondria successfully adapt to maintain energy production in response to repeated bouts of low glucose availability. It is possible that the relatively acute reductions in glucose availability in our experiments were a mild stress, insufficient to cause gross changes in mitochondrial morphology. Moreover, cells were recovered into solutions containing serum, likely providing sufficient insulin/growth factor receptor activation to aid recovery from the acute stress. Therefore, it is possible that reductions in insulin receptor activation, rather than just intracellular glucose deprivation per se, may mediate the altered mitochondrial morphology reported previously. A similar 

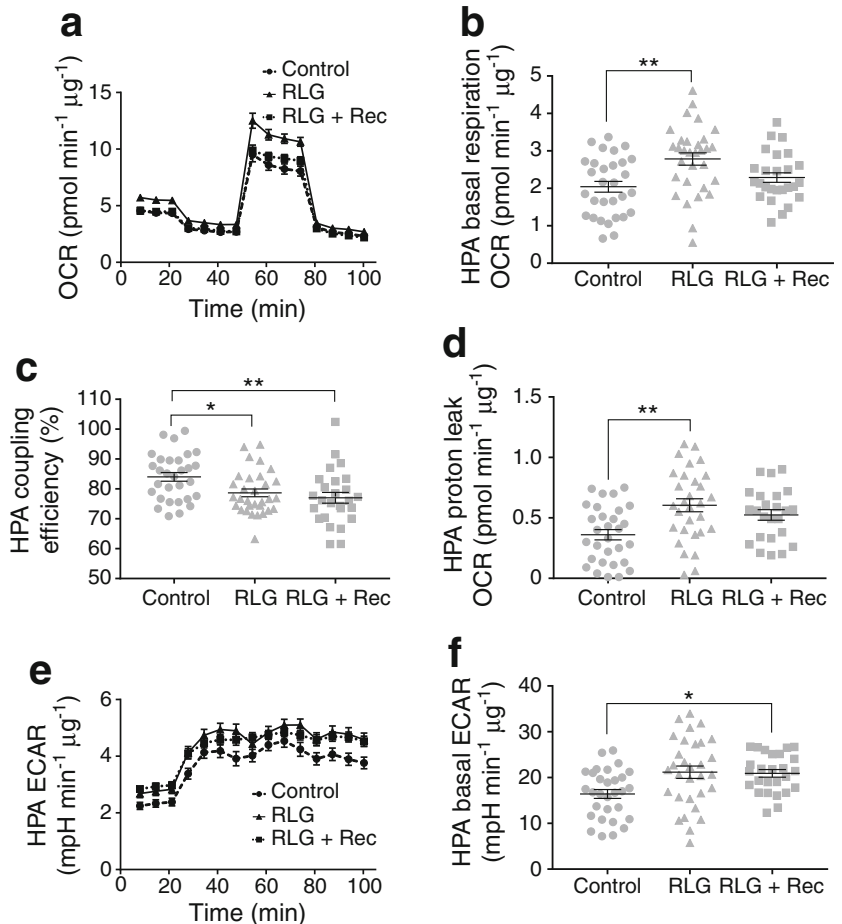

Fig. 7 Maintaining glucose levels following RLG partially recovered metabolic adaptations. (a) OCR of HPA following RLG with a 4 day recovery after the last low glucose exposure (control, $n=31$; RLG, $n=$ 31 ; RLG + recovery, $n=26)$. Cells were exposed to oligomycin $(10 \mu \mathrm{mol} / 1)$, FCCP $(5 \mu \mathrm{mol} / 1)$ and a combination of rotenone and antimycin A $(5 \mu \mathrm{mol} / \mathrm{l})$. (b) Mean basal respiration rate (OCR). (c) Coupling efficiency calculated from the ratio of oligomycin-sensitive OCR and basal OCR expressed as a percentage. (d) Proton leak, calculated from the oligomycin-insensitive (i.e. not ATP synthase-linked) OCR minus non-mitochondrial respiration, from HPA cells; RLG + recovery was not significant vs control or vs RLG. (e) ECAR analysis measured during mitochondrial stress tests (control, $n=31$; RLG, $n=31$; RLG + recovery, $n=26$ ). (f) Mean baseline ECAR in HPA cells following RLG and RLG + recovery. Error bars represent standard error of the mean. $* p<0.05, * * p<0.01$ vs control. Analyses used unpaired two-tailed Students $t$ tests. RLG + Rec, RLG + recovery

phenotype of increased mitochondrial fragmentation was also reported using inducible knockdown of insulin-like growth factor 1 (IGF-1) receptors on astrocytes [31], suggesting an important role for hormones in regulating glial cell mitochondrial function that requires further investigation.

Previous studies in rats found no change in brain glycogen levels following recurrent hypoglycaemia [32] or any correlation between brain glycogen content and impaired awareness of hypoglycaemia in humans [33]. However, other studies suggest brain glycogen content is increased following recovery from insulin-induced hypoglycaemia [34] and following recurrent 2-deoxyglucose-induced glucoprivation (reduction in utilisable glucose) in mice [35]. Our data clearly demonstrate that astrocytes intrinsically adapt by increasing cellular metabolism in response to recurrent energy stress. Specifically, rates of extracellular acidification (a marker for glycolysis) following glucose 'reperfusion' were elevated in cells exposed to RLG, suggesting an augmented re-activation of glycolysis. However, despite glycolysis being a major determinant of glycogen synthesis, we found no significant change in astrocytic glycogen levels following RLG. Extracellular lactate, which can be generated from glycogen breakdown during aglycaemia [17], increased substantially, relative to glucose availability, during low glucose exposure, though the magnitude of this response was not significantly altered following RLG. We cannot rule out, however, the possibility that more subtle changes in lactate and glycogen occur during the transition to low glucose or on glucose recovery. Moreover, before definitively ruling out a role for astrocytic glycogen and subsequent lactate release, a more replete in vitro model, containing both astrocytes and neurons, should be tested, thus facilitating the inclusion of neurotransmitter dynamics in the model. The ability to produce increasingly credible human neurons and astrocytes from inducible pluripotent stem cell (iPSC) sources could potentially enable such investigations to be performed in an in vitro human system, overcoming some of the physiological limitations of mono-culture systems.

Astrocytes play a key role in maintaining glutamatergic neurotransmission through the glutamate-glutamine cycle (for recent review see Schousboe [36]). Importantly, astrocytic glutamate clearance requires co-transport of three $\mathrm{Na}^{+}$ions [37], meaning that, via the ATP-dependent $\mathrm{Na}^{+} / \mathrm{K}^{+}$ATPase, there is a significant metabolic cost for astrocytes to maintain their $\mathrm{Na}^{+}$homeostasis, estimated to account for approximately $20 \%$ of total astrocytic ATP production [38]. This process may become particularly costly during hypoglycaemia. Moreover, glutamate stimulation of astrocytes produces mitochondrial dysfunction, decreasing mitochondrial spare respiratory capacity and increasing lactate production [39]. Furthermore, recent observations suggest that astrocytic glutamate recycling is diminished following recurrent hypoglycaemia, a change most likely mediated by reduced astrocyte glutamate uptake [40]. Interestingly, recurrent hypoglycaemia is associated with relative GABA excess within the VMH [41]. In addition to glutamate, astrocytes also clear GABA from the extracellular space [42], which is energetically less expensive for the astrocytes than glutamate recycling [43]. This raises the interesting possibility that the glutamatergic failure and relative GABA excess following recurrent hypoglycaemia may be mediated by astrocytes, plausibly as a protective mechanism against excitotoxicity, although further study is required.

Our data add to published findings and suggest that metabolic changes within astrocytes could be protective adaptations to limit brain cell damage or contribute to defective counterregulatory responses following RH. In support of the latter, astrocytes are increasingly recognised as playing an important role in the regulation of whole-body metabolism. For example, chemogenetic activation (by increasing intracellular $\mathrm{Ca}^{2+}$ ) of astrocytes in the arcuate nucleus increases feeding in mice and, conversely, sequestering astrocytic $\mathrm{Ca}^{2+}$ decreases 
food intake [14]. Moreover, astrocytes can be a source of proinflammatory cytokines in the brain [44], including TNF $\alpha$, which has previously been reported to blunt glucose-sensing glucose-inhibited neurons [45], suggesting that proinflammatory signals from astrocytes may regulate energysensing neurons of the hypothalamus [44]. Accordingly, modulation of inflammatory signalling in astrocytes modulates feeding in response to a high-fat diet [46]. Within the hindbrain, astrocytes can detect hypoglycaemia to regulate adjacent neurons to increase gastric emptying in response to a glucoprivic stimulus [13]. Astrocytes have also been shown to decrease hyperglycaemia in a rodent model of type 1 diabetes [47], suggesting that astrocytes may play fundamental roles in regulating whole-body glucose levels. It should also be noted that there is significant heterogeneity in astrocytes across different brain regions. For example, hypothalamic astrocytes express more genes for lipid oxidation compared with cortical astrocytes, which express more genes for lipid synthesis [48]. Regional differences in astrocytic glycogen content also exist, with high levels in hippocampus and cortex and comparatively lower levels in subcortical structures [49]. Within the hindbrain, only a subset of astrocytes respond to a glucoprivic stimulus [50], indicating that not all astrocytes will respond to a given stimulus in the same manner. Given the significant heterogeneity of neurons within the hypothalamus, it is plausible that subtypes of astrocytes within this region may contribute distinct roles in hypoglycaemia sensing. However, the territory of a single astrocyte is likely to overlap with multiple neuronal types, probably requiring subcellular site-specific responses.

In conclusion, our data further support the evidence that glial cells detect hypoglycaemic stress. Moreover, we demonstrate that mitochondrial function (increased FAO) in human astrocytes is altered by RLG. These data suggest a possible role for glial mitochondrial adaptations in defective glucose counterregulation or protection against hypoglycaemia, and highlight the need for more investigation into neuron-glial interactions during and following recurrent hypoglycaemia.

Acknowledgements We thank the family of the donor for making this research possible and N. Gutowski and J. Holley (both University of Exeter, Exeter, UK) for kindly gifting the human primary astrocytes. We also wish to thank N. Howe (Seahorse Bioscience, Agilent, UK) for guidance with extracellular flux analysis.

Parts of this study were presented at the American Diabetes Association 77th Scientific Sessions and at the Diabetes UK Annual Professional Conference 2017 and 2018.

Data availability The datasets generated during and analysed during the current study are available from the corresponding author on reasonable request.

Funding This study was funded by grants from: Diabetes UK (RD Lawrence Fellowship to CB; 13/0004647); the Medical Research Council (MR/N012763/1) to KLJE, ADR and CB; and a Mary Kinross Charitable Trust PhD studentship to CB, ADR and RW to support PGWP.
Additional support for this work came from awards from the British Society for Neuroendocrinology (to CB and KLJE), the Society for Endocrinology (CB), Tenovus Scotland (CB) and the University of Exeter Medical School (CB and KLJE). AR was also supported by a Royal Society Industry Fellowship.

Duality of interest The authors declare that there is no duality of interest associated with this manuscript.

Contribution statement PGWP, JMVW and JLR contributed to data acquisition. PGWP, JMVW, JLR, JKC, RW, ADR, KLJE and CB contributed to data analysis and interpretation. All authors contributed to drafting and revising the article and gave approval for the final version to be published. $\mathrm{CB}$ conceived the study and is the guarantor of this work and, as such, had full access to all the data in the study and takes responsibility for the integrity of the data and the accuracy of the data analysis.

Open Access This article is distributed under the terms of the Creative Commons Attribution 4.0 International License (http:// creativecommons.org/licenses/by/4.0/), which permits unrestricted use, distribution, and reproduction in any medium, provided you give appropriate credit to the original author(s) and the source, provide a link to the Creative Commons license, and indicate if changes were made.

\section{References}

1. Michaud I, Henderson M, Legault L, Mathieu ME (2017) Physical activity and sedentary behavior levels in children and adolescents with type 1 diabetes using insulin pump or injection therapy - the importance of parental activity profile. J Diabetes Complicat 31 : 381-386. https://doi.org/10.1016/j.jdiacomp.2016.11.016

2. Brazeau AS, Rabasa-Lhoret R, Strychar I, Mircescu H (2008) Barriers to physical activity among patients with type 1 diabetes. Diabetes Care 31:2108-2109. https://doi.org/10.2337/dc08-0720

3. Robinson RT, Harris ND, Ireland RH, Macdonald IA, Heller SR (2004) Changes in cardiac repolarization during clinical episodes of nocturnal hypoglycaemia in adults with type 1 diabetes. Diabetologia 47:312-315. https://doi.org/10.1007/s00125-0031292-4

4. Chow E, Bernjak A, Williams S et al (2014) Risk of cardiac arrhythmias during hypoglycemia in patients with type 2 diabetes and cardiovascular risk. Diabetes 63:1738-1747. https://doi.org/10. 2337/db13-0468

5. Heller SR, Cryer PE (1991) Reduced neuroendocrine and symptomatic responses to subsequent hypoglycemia after 1 episode of hypoglycemia in nondiabetic humans. Diabetes 40:223-226. https://doi.org/10.2337/diab.40.2.223

6. Segel SA, Paramore DS, Cryer PE (2002) Hypoglycemiaassociated autonomic failure in advanced type 2 diabetes. Diabetes 51:724-733. https://doi.org/10.2337/diabetes.51.3.724

7. Evans ML, McCrimmon RJ, Flanagan DE et al (2004) Hypothalamic ATP-sensitive K + channels play a key role in sensing hypoglycemia and triggering counterregulatory epinephrine and glucagon responses. Diabetes 53:2542-2551. https://doi.org/ 10.2337/diabetes.53.10.2542

8. Horvath TL, Sarman B, García-Cáceres C et al (2010) Synaptic input organization of the melanocortin system predicts dietinduced hypothalamic reactive gliosis and obesity. Proc Natl Acad Sci 107:14875-14880. https://doi.org/10.1073/pnas.1004282107

9. Mancardi GL, Liwnicz BH, Mandybur TI (1983) Fibrous astrocytes in Alzheimer's disease and senile dementia of Alzheimer's type. Acta Neuropathol 61:76-80. https://doi.org/10.1007/BF00688390 
10. Renkawek K, Stege GJ, Bosman GJ (1999) Dementia, gliosis and expression of the small heat shock proteins hsp27 and alpha Bcrystallin in Parkinson's disease. Neuroreport 10:2273-2276. https://doi.org/10.1097/00001756-199908020-00009

11. Lee J, Hyeon SJ, Im H, Ryu H, Kim Y, Ryu H (2016) Astrocytes and microglia as non-cell autonomous players in the pathogenesis of ALS. Experimental neurobiology 25:233-240. https://doi.org/ 10.5607/en.2016.25.5.233

12. Maxwell K, Berliner JA, Cancilla PA (1989) Stimulation of glucose analogue uptake by cerebral microvessel endothelial cells by a product released by astrocytes. J Neuropathol Exp Neurol 48:6980. https://doi.org/10.1097/00005072-198901000-00006

13. McDougal DH, Viard E, Hermann GE, Rogers RC (2013) Astrocytes in the hindbrain detect glucoprivation and regulate gastric motility. Autonomic neuroscience: basic \& clinical 175:61-69. https://doi.org/10.1016/j.autneu.2012.12.006

14. Chen N, Sugihara H, Kim J et al (2016) Direct modulation of GFAP-expressing glia in the arcuate nucleus bi-directionally regulates feeding. eLife 5:e18716. https://doi.org/10.7554/eLife.18716

15. Fuente-Martin E, Garcia-Caceres C, Granado M et al (2012) Leptin regulates glutamate and glucose transporters in hypothalamic astrocytes. J Clin Invest 122:3900-3913. https://doi.org/10.1172/ JCI64102

16. Wender R, Brown AM, Fern R, Swanson RA, Farrell K, Ransom BR (2000) Astrocytic glycogen influences axon function and survival during glucose deprivation in central white matter. J Neurosci 20:6804-6810. https://doi.org/10.1523/JNEUROSCI.20-1806804.2000

17. Brown AM, Sickmann HM, Fosgerau K et al (2005) Astrocyte glycogen metabolism is required for neural activity during aglycemia or intense stimulation in mouse white matter. J Neurosci Res 79:74-80. https://doi.org/10.1002/jnr.20335

18. Gibbs ME, Hertz L (2008) Inhibition of astrocytic energy metabolism by D-lactate exposure impairs memory. Neurochem Int 52 : 1012-1018. https://doi.org/10.1016/j.neuint.2007.10.014

19. Suzuki A, Stern SA, Bozdagi O et al (2011) Astrocyte-neuron lactate transport is required for long-term memory formation. Cell 144:810-823. https://doi.org/10.1016/j.cell.2011.02.018

20. Matsui $T$, Ishikawa $T$, Ito $H$ et al (2012) Brain glycogen supercompensation following exhaustive exercise. J Physiol 590: 607-616. https://doi.org/10.1113/jphysiol.2011.217919

21. Almeida A, Moncada S, Bolanos JP (2004) Nitric oxide switches on glycolysis through the AMP protein kinase and 6phosphofructo-2-kinase pathway. Nat Cell Biol 6:45-51. https:// doi.org/10.1038/ncb1080

22. Holley JE, Gveric D, Whatmore JL, Gutowski NJ (2005) Tenascin $\mathrm{C}$ induces a quiescent phenotype in cultured adult human astrocytes. Glia 52:53-58. https://oi.org/10.1002/glia.20231

23. Vlachaki Walker JM, Robb JL, Cruz AM et al (2017) AMPactivated protein kinase (AMPK) activator A-769662 increases intracellular calcium and ATP release from astrocytes in an AMPKindependent manner. Diabetes Obes Metab 19:997-1005. https:// doi.org/10.1111/dom.12912

24. Allaman I, Pellerin L, Magistretti PJ (2004) Glucocorticoids modulate neurotransmitter-induced glycogen metabolism in cultured cortical astrocytes. J Neurochem 88:900-908

25. McCrimmon RJ, Shaw M, Fan X et al (2008) Key role for AMPactivated protein kinase in the ventromedial hypothalamus in regulating counterregulatory hormone responses to acute hypoglycemia. Diabetes 57:444 450. https://doi.org/10.2337/db07-0837

26. Claret M, Smith MA, Batterham RL et al (2007) AMPK is essential for energy homeostasis regulation and glucose sensing by POMC and AgRP neurons. J Clin Invest 117:2325-2336. https://doi.org/ 10.1172/JCI31516

27. St-Pierre J, Buckingham JA, Roebuck SJ, Brand MD (2002) Topology of superoxide production from different sites in the mitochondrial electron transport chain. J Biol Chem 277:4478444790. https://doi.org/10.1074/jbc.M207217200

28. Skulachev VP (1998) Uncoupling: new approaches to an old problem of bioenergetics. Biochimica et Biophysica Acta (BBA) Bioenergetics 1363:100-124. https://doi.org/10.1016/S00052728(97)00091-1

29. Poplawski MM, Mastaitis JW, Mobbs CV (2011) Naloxone, but not valsartan, preserves responses to hypoglycemia after antecedent hypoglycemia. Diabetes 60:39-46. https://doi.org/10.2337/db100326

30. García-Cáceres C, Quarta C, Varela L et al (2016) Astrocytic insulin signaling couples brain glucose uptake with nutrient availability. Cell 166:867-880. https://doi.org/10.1016/j.cell.2016.07.028

31. Logan S, Pharaoh GA, Marlin MC et al (2018) Insulin-like growth factor receptor signaling regulates working memory, mitochondrial metabolism, and amyloid-beta uptake in astrocytes. Mol Metab 9: 141-155. https://doi.org/10.1016/j.molmet.2018.01.013

32. Herzog RI, McNay EC, McCrimmon R et al (2006) Effect of acute and recurrent hypoglycemia on regional changes in brain glycogen concentration. Diabetes 55:A149-A149

33. Oz G, Tesfaye N, Kumar A, Deelchand DK, Eberly LE, Seaquist ER (2012) Brain glycogen content and metabolism in subjects with type 1 diabetes and hypoglycemia unawareness. J Cereb Blood Flow Metab 32:256-263. https://doi.org/10.1038/jcbfm.2011.138

34. Canada SE, Weaver SA, Sharpe SN, Pederson BA (2011) Brain glycogen supercompensation in the mouse after recovery from insulin-induced hypoglycemia. J Neurosci Res 89:585-591. https://doi.org/10.1002/jnr.22579

35. Alquier T, Kawashima J, Tsuji Y, Kahn BB (2007) Role of hypothalamic adenosine $5^{\prime}$-monophosphate activated protein kinase in the impaired counterregulatory response induced by repetitive neuroglucopenia. Endocrinology 148:1367-1375. https://doi.org/ 10.1210/en.2006-1039

36. Schousboe A (2018) Metabolic signaling in the brain and the role of astrocytes in control of glutamate and GABA neurotransmission. Neurosci Lett. https://doi.org/10.1016/j.neulet.2018.01.038

37. Levy LM, Warr O, Attwell D (1998) Stoichiometry of the glial glutamate transporter GLT-1 expressed inducibly in a Chinese hamster ovary cell line selected for low endogenous $\mathrm{Na}^{+}$-dependent glutamate uptake. J Neurosci 18:9620-9628. https://doi.org/10. 1523/JNEUROSCI.18-23-09620.1998

38. Silver IA, Erecinska M (1997) Energetic demands of the $\mathrm{Na}^{+} /$ $\mathrm{K}^{+}$ATPase in mammalian astrocytes. Glia 21:35-45. https:// doi.org/10.1002/(SICI)1098-1136(199709)21:1<35::AIDGLIA4>3.0.CO;2-0

39. Yan X, Shi ZF, Xu LX et al (2017) Glutamate impairs mitochondria aerobic respiration capacity and enhances glycolysis in cultured rat astrocytes. Biomed Environ Sci 30:44-51. https://doi.org/10.3967/ bes 2017.005

40. Chowdhury GMI, Wang P, Ciardi A et al (2017) Impaired glutamatergic neurotransmission in the VMH may contribute to defective counterregulation in recurrently hypoglycemic rats. Diabetes 66:1979-1989. https://doi.org/10.2337/db16-1589

41. Chan O, Cheng H, Herzog R et al (2008) Increased GABAergic tone in the ventromedial hypothalamus contributes to suppression of counterregulatory responses after antecedent hypoglycemia. Diabetes 57:1363-1370. https://doi.org/10.2337/db07-1559

42. Iversen LL, Neal MJ (1968) The uptake of $\left[{ }^{3} \mathrm{H}\right] \mathrm{GABA}$ by slices of rat cerebral cortex. J Neurochem 15(10):1141-1149. https://doi.org/ 10.1111/j.1471-4159.1968.tb06831.x

43. Chatton J-Y, Pellerin L, Magistretti PJ (2003) GABA uptake into astrocytes is not associated with significant metabolic cost: implications for brain imaging of inhibitory transmission. Proc Natl Acad Sci 100:12456-12461. https://doi.org/10.1073/pnas. 2132096100 
44. Chung IY, Benveniste EN (1990) Tumor necrosis factor-alpha production by astrocytes. Induction by lipopolysaccharide, IFN-gamma, and IL-1 beta. J Immunol 144:2999-3007

45. Hao L, Sheng Z, Potian J, Deak A, Rohowsky-Kochan C, Routh VH (2016) Lipopolysaccharide (LPS) and tumor necrosis factor alpha $(\mathrm{TNF} \alpha)$ blunt the response of neuropeptide Y/agouti-related peptide (NPY/AgRP) glucose inhibited (GI) neurons to decreased glucose. Brain Res 1648:181-192. https://doi.org/10.1016/j. brainres.2016.07.035

46. Buckman LB, Thompson MM, Lippert RN, Blackwell TS, Yull FE, Ellacott KL (2015) Evidence for a novel functional role of astrocytes in the acute homeostatic response to high-fat diet intake in mice. Mol Metab 4:58-63. https://doi.org/10.1016/j.molmet.2014.10.001

47. Chari M, Yang CS, Lam CKL et al (2011) Glucose transporter-1 in the hypothalamic glial cells mediates glucose sensing to regulate glucose production in vivo. Diabetes 60:1901-1906. https://doi. org $/ 10.2337 / \mathrm{db} 11-0120$

48. Boisvert MM, Erikson GA, Shokhirev MN, Allen NJ (2018) The aging astrocyte transcriptome from multiple regions of the mouse brain. Cell Rep 22:269-285. https://doi.org/10.1016/j.celrep.2017. 12.039

49. Oe Y, Baba O, Ashida H, Nakamura KC, Hirase H (2016) Glycogen distribution in the microwave-fixed mouse brain reveals heterogeneous astrocytic patterns. Glia 64:1532-1545. https://doi. org/10.1002/glia.23020

50. McDougal DH, Hermann GE, Rogers RC (2013) Astrocytes in the nucleus of the solitary tract are activated by low glucose or glucoprivation: evidence for glial involvement in glucose homeostasis. Front Neurosci 7:249 